\title{
Jacques Anquetil, Anquetil-Duperron. Premier orientaliste français
}

\section{Simón Gallegos Gabilondo}

\section{(2) OpenEdition \\ 1 Journals}

\section{Edizione digitale}

URL: http://journals.openedition.org/studifrancesi/8932

DOI: 10.4000/studifrancesi.8932

ISSN: 2421-5856

\section{Editore}

Rosenberg \& Sellier

\section{Edizione cartacea}

Data di pubblicazione: 1 octobre 2008

Paginazione: 461-462

ISSN: 0039-2944

\section{Notizia bibliografica digitale}

Simón Gallegos Gabilondo, «Jacques Anquetil, Anquetil-Duperron. Premier orientaliste français», Studi Francesi [Online], 155 (LII | II) | 2008, online dal 30 novembre 2015, consultato il 12 janvier 2021. URL: http://journals.openedition.org/studifrancesi/8932 ; DOI: https://doi.org/10.4000/studifrancesi.8932

Questo documento è stato generato automaticamente il 12 janvier 2021.

\section{(c) (i) (9)}

Studi Francesi è distribuita con Licenza Creative Commons Attribuzione - Non commerciale - Non opere derivate 4.0 Internazionale. 


\title{
Jacques Anquetil, Anquetil-Duperron. Premier orientaliste français
}

\author{
Simón Gallegos Gabilondo
}

\section{NOTIZIA}

JACQUES ANQUETIL, Anquetil-Duperron. Premier orientaliste français, Paris, Presses de la Renaissance, 2006, pp. 270.

1 I non molti studiosi che si sono occupati di Abraham Hyacinthe Anquetil-Duperron (1731-1805) si sono trovati spesso in difficoltà nel dire chi egli sia stato esattamente. I più hanno visto in lui il primo orientalista francese ad aver tradotto e pubblicato in Europa i testi dello zoroastrismo e dell'induismo. Ma Anquetil-Duperron è stato anche un pioniere nel campo della filologia e dell'etnologia, oltre che dell'orientalismo. Ognuna di queste discipline trova nelle opere dello studioso settecentesco una più o meno giustificata anticipazione, anche se la ricerca delle paternità di cui Anquetil è in qualche modo responsabile, da Schopenhauer ai critici dell'etnocentrismo europeo, porterebbe troppo lontano. Jacques Anquetil, un suo lontano discendente, gli ha dedicato una biografia romanzata, che può suscitare l'attenzione del lettore anche non specialista per una personalità indubbiamente di grande interesse, pur se ancora troppo poco conosciuta. Concepita come un romanzo, la biografia non segue l'ordine cronologico.

2 La prima parte si occupa della vita di Anquetil-Duperron a partire dal momento in cui nel 1762 egli rientrò in Francia dal suo viaggio in Oriente, per il quale era partito otto anni prima. Vi si narra del suo incontro con i suoi familiari e con i protettori dell'Académie des Inscriptions et Belles-Lettres (di cui farà parte) e si accenna all'importanza che il suo primo lavoro, lo Zend-Avesta (pubblicato nel 1771), ebbe per gli sviluppi dell'orientalismo e per l'opera di studiosi come Eugène Burnouf, James Darmesteter e Silvestre de Sacy. Vengono toccate anche tematiche di interesse storiografico che sollevano la questione dell'oblio in cui si trova oggi Anquetil- 
Duperron come autore, aspetto legato alla diffusione di una sua immagine negativa ad opera di alcuni intellettuali del tempo. Parte del discredito di cui soffre ancora Anquetil-Duperron risale infatti alla ricezione da parte dei philosophes proprio dello Zend-Avesta, ovvero della traduzione dei testi attribuiti a Zoroastro che AnquetilDuperron aveva raccolto durante il suo viaggio, preceduta da un importante Discours préliminaire. Gli ambienti colti dell'epoca misero in dubbio l'autenticità dei manoscritti raccolti dal viaggiatore in India, polemizzando sull'utilità dei lunghi e difficili anni da lui passati alla loro ricerca. Anquetil-Duperron venne ridicolizzato in particolare da alcune personalità importanti come Voltaire, Jacob Grimm e William Jones, con il risultato che il suo lavoro non fu considerato dai contemporanei come avrebbe meritato e fu presto dimenticato dai posteri.

3 La seconda parte del libro di J. Anquetil è dedicata agli anni trascorsi in India, ricostruiti dall'autore sulla base del racconto autobiografico che ne lasciò il suo antenato. All'origine di questa avventura vi è la scoperta, fatta da Anquetil-Duperron, allora ventitreenne, alla biblioteca del re, di alcuni fogli manoscritti in lingua zend, o avestica, calcati dagli originali conservati ad Oxford, che nessun europeo era riuscito fino a quel momento a leggere. Il giovane Anquetil-Duperron, impulsivamente, e senza avvisare né parenti né amici, decise di arruolarsi come soldato nella Compagnia francese delle Indie allo scopo di imparare sul posto la lingua degli antichi testi di Zoroastro. Trascorse lunghi anni in città e villaggi dell'India, da Pondichéry a Chandernagor fino a Surat, dove diventò discepolo dei Parsi, depositari della dottrina del profeta iraniano, che gli insegnarono la loro antica lingua sacra e lo aiutarono nella comprensione e nella traduzione dei testi.

4 La terza ed ultima parte si occupa della maturità del nostro viaggiatore, della sua evoluzione intellettuale e della pubblicazione delle altre sue opere. Negli ultimi anni della sua esistenza, Anquetil-Duperron dedicò maggiore attenzione ai problemi sollevati dalla colonizzazione, di cui egli era stato testimone durante gli anni del suo viaggio. La fase di pensiero anticolonialista ed egualitario che lo avrebbe accompagnato fino alla fine dei suoi giorni, iniziò con la pubblicazione, nel 1778, della Législation orientale, opera che confutava le tesi di Montesquieu sul dispotismo orientale; essa si espresse anche in altre opere nelle quali, senza mai smettere i panni dell'orientalista (nel 1801-1802 pubblicò l'Oupnek'hat, traduzione latina di una versione persiana di alcune Upanishad), Anquetil-Duperron denunciò gli abusi del colonialismo europeo. In quegli anni lo studioso adottò uno stile di vita molto ritirata, quello del renonçant, o dello «yoghi cristiano» come è stato scritto, vivendo in un isolamento ascetico e in una povertà volontaria, che lo portò a rifiutare gli aiuti economici di familiari ed amici, e perfino una pensione conferitagli dal governo.

5 Oltre che sul Discours préliminaire che precede lo Zend-Avesta, la biografia di Jacques Anquetil è basata su una parte importante della letteratura critica a disposizione, tra cui i lavori di Raymond Schwab, Jean-Luc Kieffer, Romain Stropetti, oltre che sui manoscritti e le lettere inedite conservate alla Bibliothèque nationale de France. Il libro di Jacques Anquetil deluderebbe i lettori che cercassero in esso un'opera accademica. Esso è, in realtà, un felice sodalizio tra la forma di scrittura adottata, quella del romanzo, e il suo contenuto, risultato di una ricerca ben documentata sulla vita dell'autore. Si tratta insomma di un'ottima introduzione alla figura di AnquetilDuperron, se non anche al suo pensiero, che rimane ancora tutto da scoprire. 